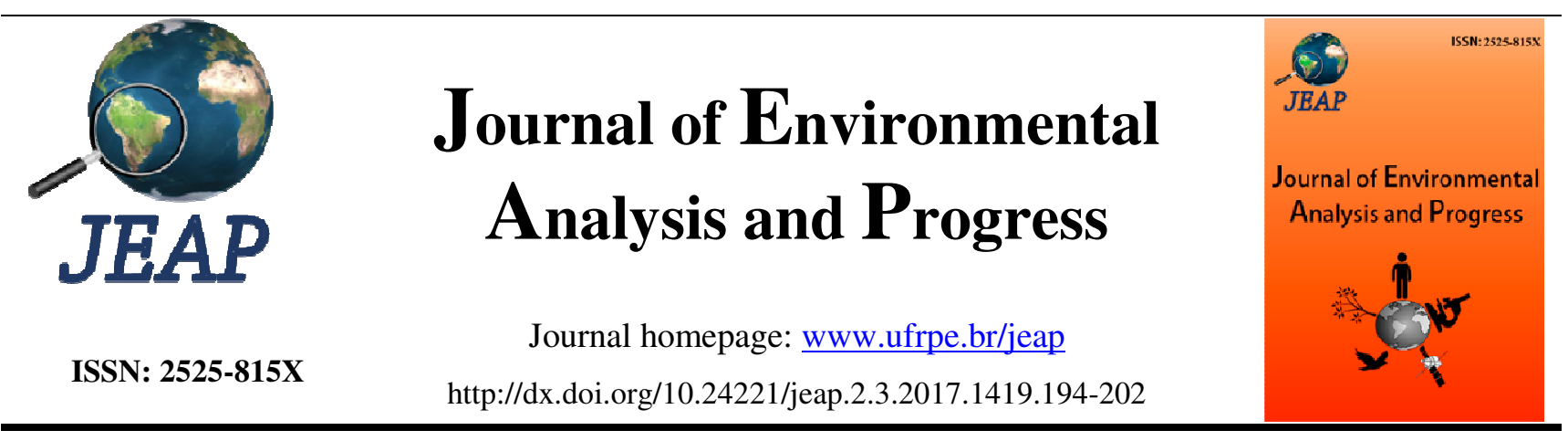

\title{
Zoneamento agrometeorológico da moringa para o Estado de Pernambuco em condições atuais e projeções futuras
}

\section{Agrometeorological zoning of moringa for the State of Pernambuco in current conditions and future projections}

\author{
Ailton Alves de Carvalho ${ }^{\mathrm{a}}$, Abelardo Antônio de Assunção Montenegro ${ }^{\mathrm{a}}$, Célia Silva dos Santos ${ }^{\mathrm{a}}$, \\ Thieres George Freire da Silva ${ }^{b}$ \\ aDepartamento de Engenharia Agrícola-DEAGRI, Universidade Federal Rural de Pernambuco-UFRPE, Rua Dom \\ Manoel de Medeiros, s/n, Dois Irmãos, Recife, Pernambuco. CEP: 52171-900. E-mail: ailtonalvesst@gmail.com; \\ abelardomontenegro666@gmail.com; celia@agro.eng.br. \\ ${ }^{b}$ Unidade Acadêmica de Serra Talhada-UAST, UFRPE, Av. Gregório Ferraz Nogueira,s/n, José Tomé de Souza Ramos, \\ Serra Talhada, Pernambuco. CEP: 56909-535. E-mail: thieres_freire@yahoo.com.br.
}

\section{A R T I C L E I N F O}

Recebido 25 Jun 2017

Aceito 02 Jul 2017

Publicado 31 Jul 2017

\begin{abstract}
A B S T R A C T
The Moringa oleifera Lam. has become, over the years, an important potential alternative in the Brazilian semi-arid, presenting high potential due to the multiplicity of uses in livestock and agriculture. Given the importance of the moringa for commercial production of products and by-products from its cultivation, its expansion in other places with characteristics favorable to its development becomes desirable. This context, the objective of this study was to perform agroclimatic zoning to identify areas of greater aptitude for moringa cultivation in the State of Pernambuco. It was verified, based on the cross-climatic information, that $39.77 \%$ of the state area presented adequate conditions for moringa cultivation in the present scenario, $79.69 \%$ in $\mathrm{B} 2$ and $98.59 \%$ in A1F1. It was found that the Applied Areas with Complementary Irrigation (AIOB) were $27.73 \%, 37.70 \%$ and $41.86 \%$ in the current scenario, B2 and A1F1, respectively. The areas totally apt to the localized cultivation, in the Sertão and in the Agreste of the State, and with the projections of climatic changes in relation to the thermal restriction will occur the increase of apt areas throughout the state. However, the zoned areas with thermal restriction do not preclude the cultivation of the moringa, but it reduces the maximum productive potential of the crop. Those with water restrictions, the incentive for the use of irrigation can favor the achievement of adequate yields.
\end{abstract}

Keywords: Climatic indicators, agroclimatology, oleaginous.

\section{R E S U M O}

A Moringa oleifera Lam. tornou-se, ao longo dos anos, uma importante alternativa potencial no semiárido brasileiro, apresentando grande potencial em face da multiplicidade de usos na pecuária e agricultura. Diante da importância da moringa para produção comercial de produtos e subprodutos oriundos do seu cultivo, a sua expansão em outros locais com características favoráveis ao seu desenvolvimento torna-se desejável. Nesse contexto, o objetivo deste estudo foi realizar o zoneamento agroclimático para identificar áreas de maior aptidão para o cultivo da moringa no Estado de Pernambuco. Constatou-se, a partir do cruzamento das informações climáticas, que $39,77 \%$ da área do estado apresentou condições adequadas ao cultivo da moringa no cenário atual, $79,69 \%$ no B2 e $98,59 \%$ no 
A1F1. Constatou-se que as áreas Aptas com Irrigação Complementar (AIOB), foram de 27,73\%, 37,70\% e 41,86\% no cenário atual, B2 e A1F1, respectivamente. As áreas totalmente aptas ao cultivo estão localizadas, no sertão e no agreste do estado, e com as projeções de mudanças climáticas em relação a restrição térmica ocorrerá o aumento de áreas aptas ao longo de todo estado. Entretanto, as áreas zoneadas com restrição térmica não impossibilita o cultivo da moringa, no entanto reduz o máximo potencial produtivo da cultura. As com restrições hídricas, o incentivo pelo uso de irrigação e reuso pode favorecer a obtenção de bons rendimentos.

Palavras-Chave: Indicadores climáticos, agroclimatologia, oleaginosa.

\section{Introdução}

Uma possível estratégia para o desenvolvimento do semiárido é o incentivo da produção de espécies que detenham importante valor econômico, a fim de gerar renda e bem-estar social para as populações (Bezerra, 2004).

Entre as espécies do semiárido com grande potencial econômico, destaca-se a Moringa oleífera Lam. A espécie é nativa da Índia, e foi introduzida no Brasil na década de 1950. É cultivada na África, Ásia, América Latina e em quase todos os países de clima tropical. As famílias que residem no semiárido nordestino têm seu sustento dependente, principalmente, de atividades de agricultura e pecuária (Santana et al., 2010).

De acordo com Oliveira et al. (2009), uma alternativa para esta região é o plantio de moringa, espécie utilizada na agricultura familiar como fonte de suplemento alimentar, uma vez que suas folhas são fontes de vitamina $A$, vitamina $B$ e $C$, aminoácidos (metionina e cisteína) e minerais, como ferro (582 mg. $\mathrm{kg}^{-1}$ ), potássio $\left(21,7 \mathrm{mg} \cdot \mathrm{kg}^{-1}\right)$, cálcio $\left(26,4 \mathrm{mg} \cdot \mathrm{kg}^{-1}\right)$ e zinco (113,9 mg. $\left.\mathrm{kg}^{-1}\right)$ (Teixeira et al., 2012).

Suas sementes também apresentam propriedades ideais para a produção de biodiesel (Monteiro, 2007; Santana et al., 2010). Alguns estudos indicam que a espécie pode produzir até 3 toneladas de semente por hectare, a qual rende cerca de $78 \%$ de óleo rico em ácido oléico. Isto indica que o produto é adequado para a obtenção de um biodiesel com baixo teor de insaturações (Guirra, 2008), que pela sua vez, é mais estável diante da oxidação, facilitando o transporte e o armazenamento.

Diante da importância da moringa para produção comercial de produtos e subprodutos oriundos do seu cultivo, a sua expansão em outros locais com características favoráveis ao seu desenvolvimento torna-se desejável. Desta forma, o zoneamento é uma das ferramentas que possibilitam a identificação dos locais mais propícios, através da delimitação visual em mapas, das áreas climaticamente adequadas com e sem restrições de cultivo (Gasparini et al., 2015). Nesse contexto, o objetivo deste estudo foi definir áreas de maior aptidão climática para o cultivo da moringa no Estado de Pernambuco por intermédio do zoneamento agroclimatológico na condição clima atual, bem como, considerando as condições climáticas futuras na região.

\section{Material e Métodos}

A área de estudo é representada pelo Estado de Pernambuco, que possui uma área territorial de $98.345,58 \mathrm{~km}^{2}$. Está localizado entre os paralelos de $7^{\circ} 18^{\prime} 17^{\prime \prime}$ a 9 $9^{\circ} 28^{\prime} 43^{\prime \prime}$ de latitude Sul e os meridianos $34^{\circ} 48^{\prime} 15^{\prime \prime}$ a $41^{\circ} 21^{\prime} 22^{\prime \prime}$ longitude Oeste de Greenwich (Silva et al., 2014). O Estado de Pernambuco apresenta 12 regiões de desenvolvimento (Metropolitana de Recife, Mata Norte, Mata Sul, Agreste Setentrional, Agreste Central, Agreste Meridional, Sertão do Moxotó, Sertão do Pajeú, Sertão de Itaparica, Sertão Central, Sertão do Araripe e Sertão do São Francisco) (Figura 1). O Estado é classificado em dois tipos climáticos segundo Köppen, em As' e BSh, com precipitações no outono e inverno em parte do litoral, e no sertão clima tropical seco no sertão com chuvas concentradas no verão e outono (Temperatura média anual $>18^{\circ} \mathrm{C}$ ).

As etapas metodológicas empregadas para elaboração do zoneamento agroclimático foram: delimitação das faixas térmicas e hídricas representativas das exigências climáticas da cultura; espacialização das características climáticas da região relacionado à deficiência hídrica anual e temperatura média anual de uma série meteorológica de 20 anos para o Estado de Pernambuco; reclassificação espacial das características climáticas da região com base nas exigências climáticas da cultura e a elaboração do mapa de zoneamento agroclimático, com delimitação das áreas em $\mathrm{km}^{2}$ e porcentagem, como realizado por Gasparini et al. (2015).

Os dados utilizados para a confecção dos mapas foram da temperatura e precipitação total anual, espacializados ao longo do Estado e depois convertidos em arquivo vetorial do tipo ponto, no formato shapefile (shp). Os dados climáticos para a confecção dos mapas foram provenientes do Departamento de Ciências Atmosféricas (DCA), obtidos em: http://www.dca.ufcg.edu.br/tsm.htm, e os dados de precipitação foram da Rede Hidrometeorológica da Superintendência de 
Desenvolvimento do Nordeste (SUDENE). As médias mensais utilizadas foram de postos pluviométricos com mais de 20 anos de dados de precipitação e temperatura do Estado de Pernambuco, totalizando, desta forma, 165 pontos de observação.

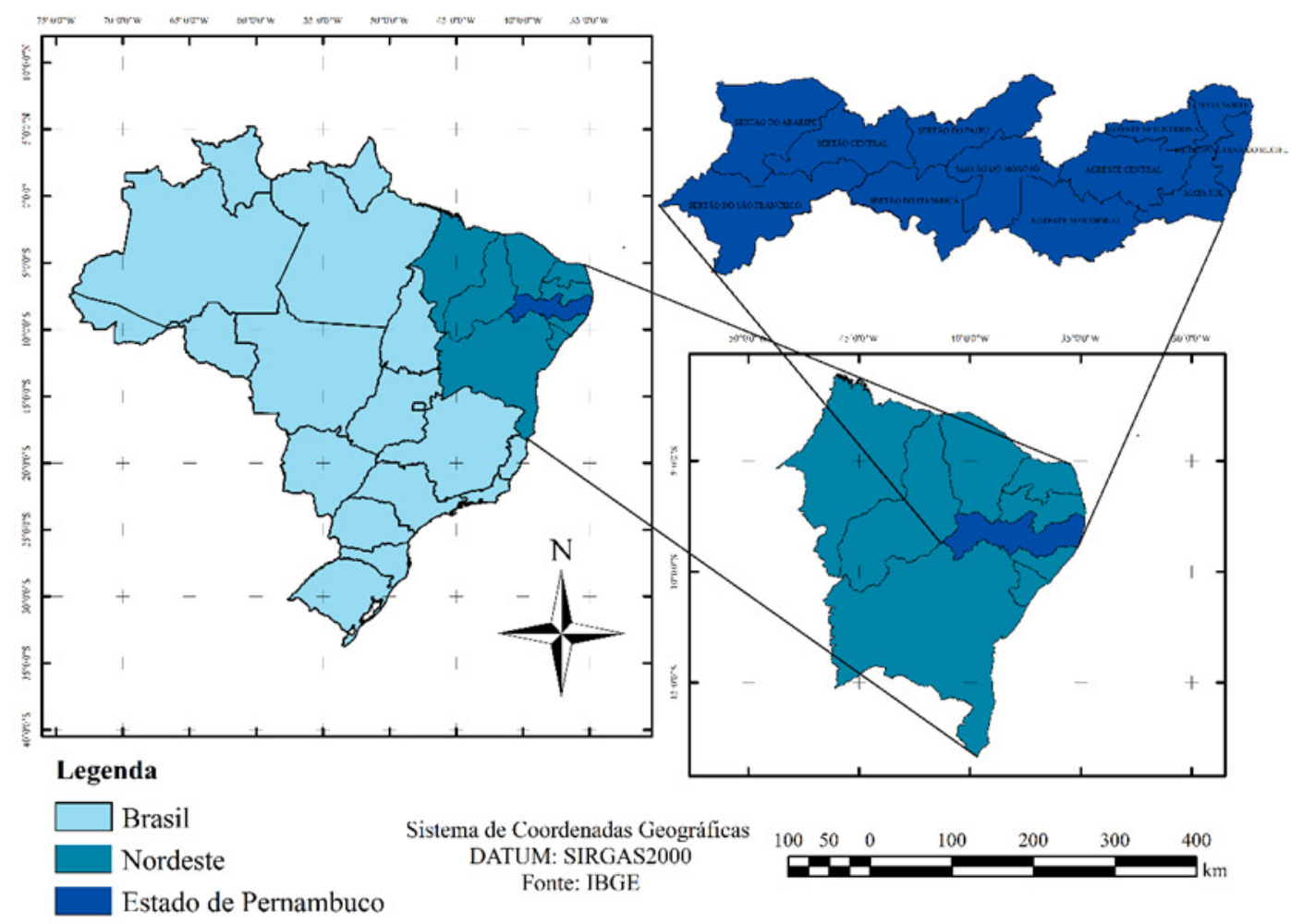

Figura 1. Localização da área de estudo - Estado de Pernambuco - no Nordeste do Brasil.

Foram considerados dois cenários de mudanças climáticas, segundo o Intergovernmental Panel on Climate Change (IPPC), simulados para o efeito na temperatura: cenário B2 - considerou-se o incremento de $1,8^{\circ} \mathrm{C}$ da temperatura e o cenário $\mathrm{A} 1 \mathrm{~F} 1$ - considerou-se o incremento de $4^{\circ} \mathrm{C}$ da temperatura (Morais et al., 2015; IPCC, 2013). Esses cenários estimam aumento na temperatura em escala global, desta forma, os incrementos foram adicionados aos valores médios atuais em escala anual para cada estação.

Em decorrência de incertezas quanto às variações previstas para a precipitação, referente aos cenários B2 e A1F1 para o Nordeste, assumiuse os valores normais atuais para cada estação, de forma inalterada até o final do século (Silva et al., 2010).

A espacialização da precipitação e da temperatura foi realizada mediante a utilização do método do inverso do quadrado da distância, pois segundo Gardiman Junior et al. (2012), é o que apresenta melhor desempenho para a estimativa espacial de dados meteorológicos.

Após a espacialização das variáveis de temperatura e precipitação, as imagens matriciais obtidas foram reclassificadas, de acordo com a exigência da cultura (Tabela 1).

Após a reclassificação, a espacialização do mapa temático para o zoneamento foi realizada pela combinação das duas imagens de aptidão, restrita ou inapta, para a temperatura no cenário atual e nos dois cenários de mudanças climáticas, combinados com a precipitação.

Após a reclassificação, a espacialização do mapa temático para o zoneamento foi realizada pela combinação das duas imagens de aptidão, restrita ou inapta para a temperatura no cenário atual e nos dois cenários de mudanças climáticas combinados com a precipitação. Deve-se ressaltar que, para locais onde a precipitação é abaixo do ideal para o cultivo, se existir aptidão para a temperatura, a produção da moringa pode ocorrer desde que seja irrigada, no entanto se a precipitação for acima do ideal a área será inapta, mesmo com a temperatura na faixa ideal. Desta forma, foram delimitadas áreas com as seguintes características: Apta Sem Irrigação (ASI); Apta com Irrigação Complementar (AICO); Inapta por Excesso de Chuva (IEC); Apta com Irrigação Obrigatória (AIOB); Restrição Térmica e Apta Sem Irrigação (RT-ASI); Restrição Térmica e 
Apta com Irrigação Complementar (RT-AICO);

Restrição Térmica e Apta com Irrigação

Obrigatória (RT-AIOB) e Restrição Térmica e Inapta por Excesso de Chuva (RT-IEC).

Tabela 1. Faixas de aptidão térmica e pluviométrica para a cultura da moringa (Moringa oleifera Lam.).

\section{Temperatura}

Precipitação

Aptidão

$\left({ }^{\circ} \mathbf{C}\right)$

Aptidão

(mm)

$\begin{array}{lccc}\text { Apta } & 25 \text { a } 35^{\circ} \mathrm{C}^{\mathrm{a}} & \text { Apta com Irrigação Obrigatória (AIOB) } & <400^{\mathrm{b},} \\ \text { Restrita } & <25^{\circ} \mathrm{C} \mathrm{e} \mathrm{de} & \text { Apta com Irrigação Complementar (AICO) } & 400 \mathrm{a} 600 \mathrm{~mm}^{\mathrm{ab}} \\ \text { Inapta } & 35 \mathrm{a} 38^{\circ} \mathrm{C}^{\mathrm{a}} & & \\ & >38^{\circ} \mathrm{C}^{\mathrm{a}} & \text { Apta Sem Irrigação (ASI) } & 600 \mathrm{a} 1200 \mathrm{~mm}^{\mathrm{a}} \\ & & \text { Inapta } & >2000 \mathrm{~mm}^{\mathrm{a}}\end{array}$

Fonte: Adaptado de (Gaza, 2007) e Cáceres et al. (1991) .

\section{Resultados}

A temperatura média anual do ar no cenário atual variam de 19,5 a $26,4^{\circ} \mathrm{C}$ (Figura $2 \mathrm{~A}$ ), no B2 de 21,3 a $28,2^{\circ} \mathrm{C}$ (Figura $2 \mathrm{~B}$ ) e $\mathrm{A} 1 \mathrm{~F} 1$ de 23,5 a $30,4^{\circ} \mathrm{C}$ (Figura $2 \mathrm{C}$ ). Verificou-se que as temperaturas mais altas acontecem no sertão do Estado, as mais amenas no agreste e em pontos com altitudes mais elevadas e as intermediárias na zona da Mata e na parte Norte no agreste e sertão. Percebe-se que, para os cenários futuros com o acréscimo de $1,8^{\circ} \mathrm{C}$ e $4^{\circ} \mathrm{C}$, ocorre grande variabilidade espacial da temperatura média do ar no Estado de Pernambuco, conforme a classificação para cruzamento e reclassificação dos mapas zoneados de acordo com a aptidão. No cenário atual a condição de temperatura adotada $\left(25^{\circ} \mathrm{C}\right.$ a $\left.38^{\circ} \mathrm{C}\right)$, promove menor área de adaptação térmica. Assim, com a elevação da temperatura, a tendência que ocorra maior adaptação, possibilitando expressão do maior potencial genético da moringa.
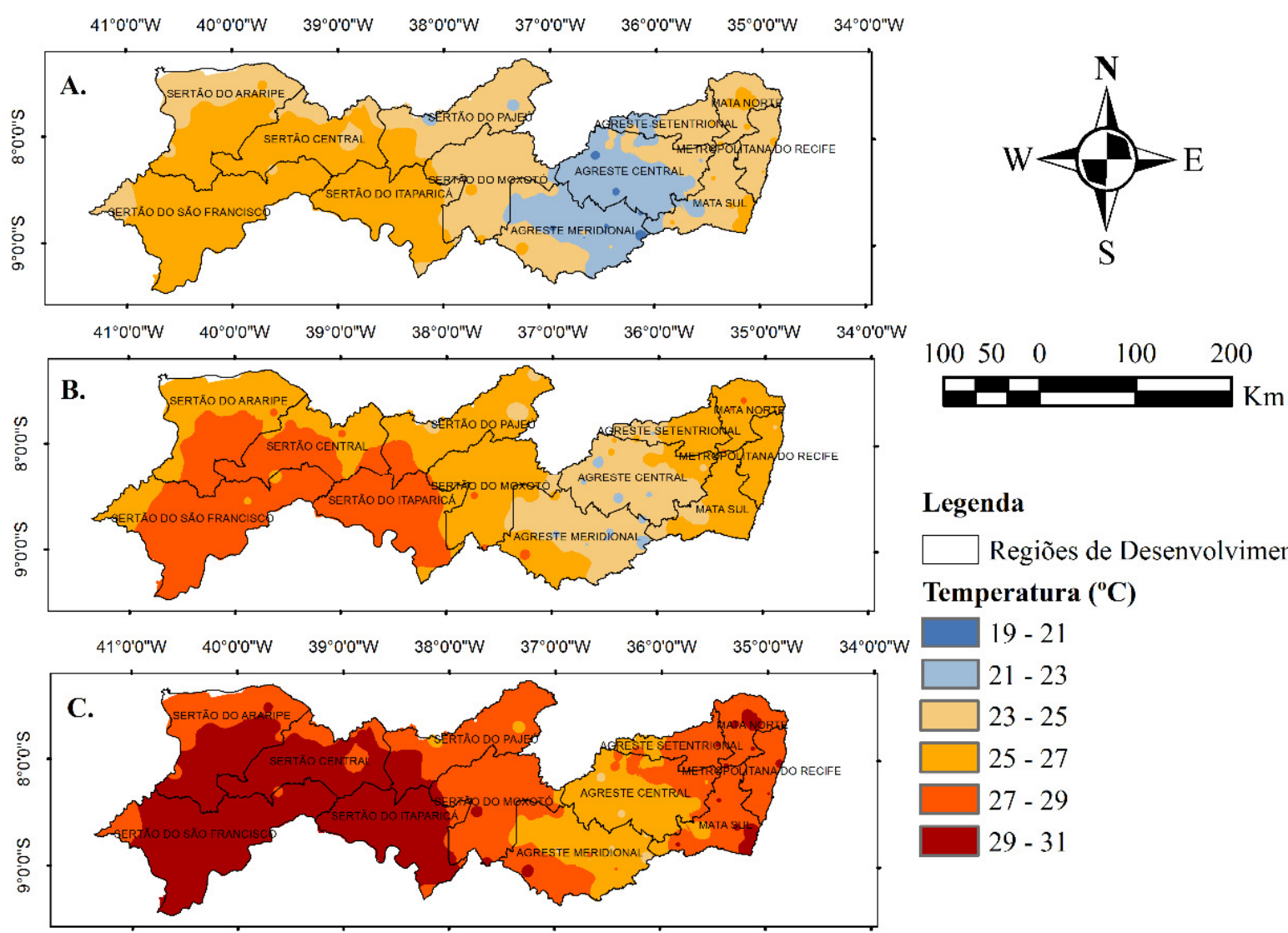

Legenda

$\square$ Regiões de Desenvolvimento Temperatura $\left({ }^{\circ} \mathrm{C}\right)$

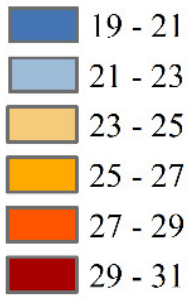

Figura 2. Mapas de temperatura no cenário atual (A), B2 (B) e A1F1 (C) para o estado de Pernambuco.

A Figura 3 mostra a delimitação da precipitação ao longo do Estado de Pernambuco, de acordo com as faixas de aptidão usadas no cruzamento e reclassificação em áreas zoneadas 
para aptidão da moringa. Pode-se observar que a faixa de precipitação de 400 a $600 \mathrm{~mm}$, ocorre na parte sul do sertão do Estado, com os menores valores de precipitação ocorrendo na mesorregião do São Francisco. A precipitação da ordem de 600 a $1200 \mathrm{~mm}$ ganha destaque no agreste pernambucano, com uma pequena porção da Zona da Mata nesta faixa. A outra faixa de aptidão, com valores de 1200 a $2500 \mathrm{~mm}$, ocorre apenas na Zona da Mata Sul e norte e na Região Metropolitana.
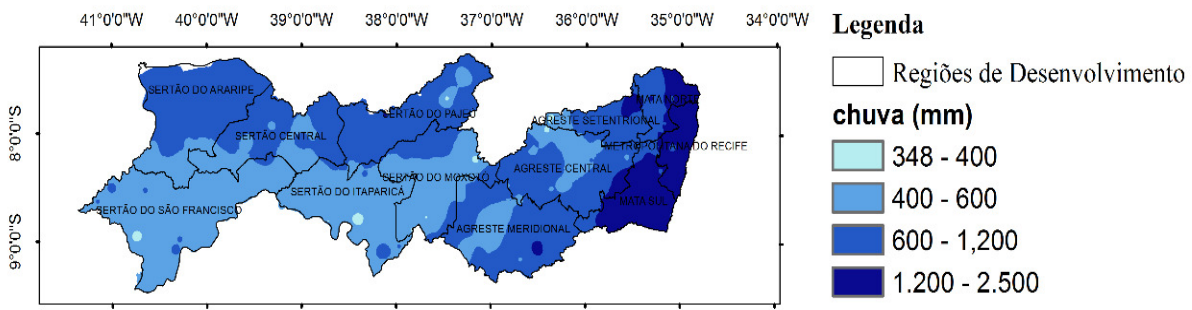

Figura 3. Mapas de precipitação, considerando as faixas de aptidão para a moringa no Estado de Pernambuco.

Oito zonas de aptidão climática foram obtidas após o cruzamento entre os mapas individuais das variáveis climáticas (temperatura e precipitação) nos cenários de mudanças climáticas, reclassificadas para atender as faixas de aptidão climática da cultura da moringa (Figura 4). As zonas foram enquadradas, especificamente para o estudo, nas classes de aptidão climática para a temperatura em aptas, restritas e inaptas, e para a precipitação em apta sem irrigação, apta com irrigação complementar, apta com irrigação obrigatória ou inapta por excesso de chuva. Com os resultados, constatou-se a existência das oito zonas no cenário atual, sendo quatro relacionada a restrição térmica, oito zonas no cenário $\mathrm{B} 2$, mais com menores áreas com restrição térmica e apenas seis zonas no cenário A1F1, com destaque apenas para duas zonas de restrições térmicas.

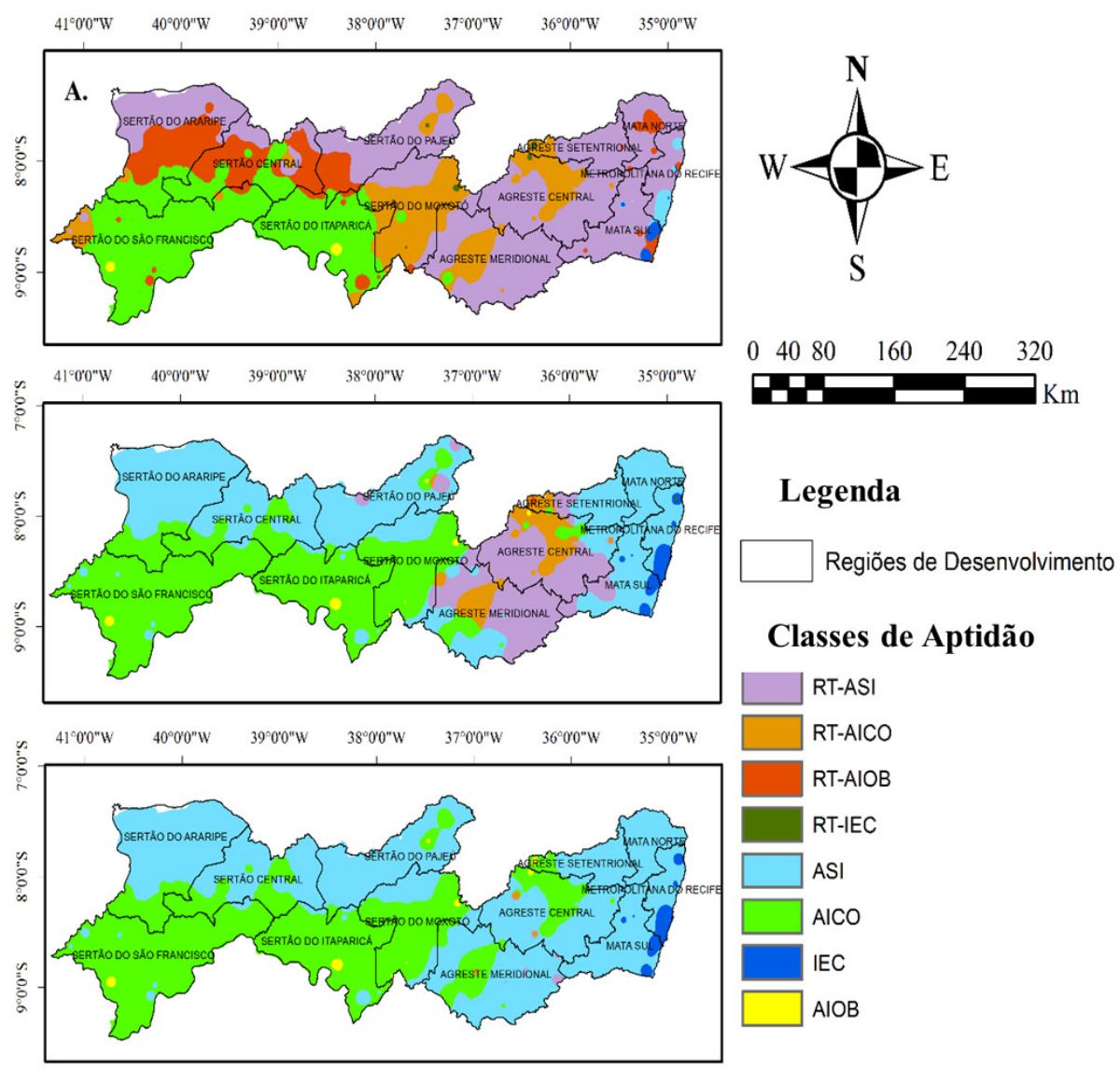

Figura 4. Classes de aptidão climática para o cultivo da moringa no Estado de Pernambuco, para o cenário atual (A), B2 (B) e A1F1 (C), com as classes: Apta Sem Irrigação (ASI); Apta com Irrigação Complementar 
(AICO); Inapta por Excesso de Chuva (IEC); Apta com Irrigação Obrigatória (AIOB); Restrição Térmica e Apta Sem Irrigação (RT-ASI); Restrição Térmica e Apta com Irrigação Complementar (RT-AICO); Restrição Térmica e Apta com Irrigação Obrigatória (RT-AIOB) e Restrição Térmica e Inapta por Excesso de Chuva (RT-IEC).

Percebe-se, para o cenário atual (Figura 4A), que a restrição térmica ocupa maior área no agreste, boa porção da parte norte do Nordeste e grandes áreas da Zona da Mata e Região Metropolitana do Estado de Pernambuco. Ao todo 60,04\% da área do estado apresenta cenários de restrição térmica nas categorias (RT-AICO, RTASI, RT-AIOB e RT-IEC). No cenário B2, as zonas com restrição térmica diminuíram para $19,16 \%$, principalmente no sertão, zona da mata e região metropolitana de recife, logo, no agreste a diminuição foi pouca (Figura 4B). O elevado valor de temperatura $4^{\circ} \mathrm{C}$, no cenário $\mathrm{A} 1 \mathrm{~F} 1$, promoveu aptidão sem restrição ao cultivo da moringa praticamente para todo o Estado, com apenas $0,31 \%$ de área restrita.

A aptidão para o cultivo da moringa se destaca na classe Apta Sem Irrigação (ASI) o que corresponde a porcentagem de $56,29 \%$ do território do Estado, caracterizada no cenário
A1F1 onde ocorre aumento de $4^{\circ} \mathrm{C}$ na temperatura atual, $41,67 \%$ no cenário $\mathrm{B} 2$, na qual a projeção do aumento da temperatura é de $1,8^{\circ} \mathrm{C}$, enquanto que apresenta apenas $11,79 \%$ de aptidão para o cenário atual (Tabela 2). Observa-se também no cenário A1F1 que a moringa é Inapta por Excesso de Chuva com percentual de $1,16 \%$ da área total, enquanto que no cenário B2 foi de $1,14 \%$ e no atual foi de $0,41 \%$.

Do total de área do estado com restrição térmica, $60,04 \%, 16,16 \%$ e $0,31 \%$, destaca-se a classe de Restrição Térmica e Apta Sem Irrigação (RT-ASI), com $44,91 \%, 14,82 \%$ e $0,20 \%$, para os cenários: atual, B2 e A1F1, respectivamente. Para as áreas zoneadas de Restrição Térmica e Apta com Irrigação Complementar (RT-AICO), da porcentagem de área total $(60,04 \%, 16,16 \%$ e $0,31 \%$ ), os valores de $14,41 \%, 4,27 \%$ e $0,11 \%$ são dos cenários: atual, B2 e A1F1, respectivamente.

Tabela 2. Área e porcentagem do zoneamento dos cenários de aptidão climática da moringa para o Estado de Pernambuco.

\begin{tabular}{|c|c|c|c|c|c|c|}
\hline \multicolumn{7}{|c|}{ Cenários } \\
\hline \multirow{2}{*}{ Classes } & Atual & B2 & A1F1 & Atual & B2 & A1F1 \\
\hline & & $\mathbf{k m}^{2}$ & & & $\%$ & \\
\hline AIOB & 219,59 & 317,76 & 372,56 & 0,22 & 0,32 & 0,38 \\
\hline AICO & 27165,37 & 37074,11 & 41169,07 & 27,68 & 37,70 & 41,86 \\
\hline ASI & 11570,65 & 40983,67 & 55355,36 & 11,79 & 41,67 & 56,29 \\
\hline IEC & 403,38 & 1124,17 & 1138,94 & 0,41 & 1,14 & 1,16 \\
\hline RT-AICO & 14111,66 & 4202,97 & 107,82 & 14,38 & 4,27 & 0,11 \\
\hline RT-ASI & 43985,98 & 14572,75 & 201,25 & 44,83 & 14,82 & 0,20 \\
\hline RT-AIOB & 152,96 & 54,76 & ----- & 0,16 & 0,06 & ----- \\
\hline RT-IEC & 735,52 & 14,77 & ----- & 0,75 & 0,02 & ----- \\
\hline
\end{tabular}

Apta Sem Irrigação (ASI); Apta com Irrigação Complementar (AICO); Inapta por Excesso de Chuva (IEC); Apta com Irrigação Obrigatória (AIOB); Restrição Térmica e Apta Sem Irrigação (RT-ASI); Restrição Térmica e Apta com Irrigação Complementar (RT-AICO); Restrição Térmica e Apta com Irrigação Obrigatória (RT-AIOB) e Restrição Térmica e Inapta por Excesso de Chuva (RT-IEC).

\section{Discussão}

Apesar da grande variabilidade de chuvas em Pernambuco, as atividades produtivas no Estado sofrem impactos adversos das condições de baixa, média ou alta pluviosidade, mas principalmente da sua variabilidade temporal e espacial (Possas et al., 2012).

As áreas situadas na classe inapta apresentam restrição forte ao cultivo, com limitação da produção devido ao excesso de água que, segundo Parrotta (1993), a moringa apresenta sensibilidade em solos muito úmidos. Essas áreas zoneadas ocorrem em parte da Zona da Mata Sul e Região Metropolitana do Recife (Figura 4), com $1,16 \%$ de área inapta.

Atualmente existem reduzidas áreas de cultivo de moringa ao longo do Estado, com esta cultura representando importante potencial de renda para a agricultura e pecuária. 
Visualmente, as áreas com irrigação complementar encontram-se no sertão do Estado, com uma pequena área no agreste, e as áreas com irrigação obrigatória é pouca ao longo do Estado, visto que a cultura produz bem na faixa de 600 a $1200 \mathrm{~mm}$. Existem relatos da produção de moringa em áreas a partir de $250 \mathrm{~mm}$ (Gaza, 2007), no entanto, sem expressar seu real potencial genético. Além do mais, pode observar o grande potencial de produção ao longo do estado, visto que a menor índice de precipitação médio anual foi de $348,8 \mathrm{~mm}$. Por outro lado, na Região de Zona da Mata e Metropolitana 11 cidades apresentaram média de precipitação acima de $2000 \mathrm{~mm}$, logo impossibilitando o cultivo.

$\mathrm{Da}$ área apta total ao cultivo da moringa $(39,77 \%, 79,69 \%$ e 98,53\%), destaca-se a área Apta com Irrigação Complementar (AIOB), com $27,73 \%, 37,70 \%$ e $41,86 \%$ no cenário atual, B2 e $\mathrm{A} 1 \mathrm{~F} 1$, respectivamente. Essas áreas foram observadas praticamente no sertão do Estado, e apenas pequenas porções no agreste. Assim, desde que o produtor tenha disponibilidade de água, seja de uma fonte natural ou por meio do reuso de água como na estação piloto de Mutuca administrada pela UFRPE, a complementação promoverá o aumento de rendimento da cultura. Entretanto, vale salientar que esta complementação hídrica deve ser uma prática a ser realizada durante o período de estiagem de cada região. Essa necessidade de suplementação hídrica, de acordo com Nobrega et al. (2015), ocorre devido a variabilidade espacial e temporal da precipitação, com destaque para o Sertão Pernambucano e do São Francisco, com ocorrência de meses extremamente secos, na qual, a irrigação complementar torna-se necessária para a maioria das culturas durante o período seco, e caso não seja feita, os impactos gerados devido a deficiência hídrica fará com que ocorra redução ou oscilação do rendimento da cultura. Ressalta-se ainda, que para uma maior eficiência no uso da água, práticas conservacionistas devem ser utilizadas para promover a permanência da umidade por maior período no solo, como a cobertura morta principalmente em condições de semiárido como destacado nos estudos de Montenegro et al. (2013), Borges et al. (2014) e Abrantes et al. (2015).

Obviamente, é importante ressaltar que, as áreas zoneadas com restrição térmica não impossibilita o cultivo da moringa, no entanto reduz o máximo potencial produtivo da cultura. Desta forma, nas áreas onde se faz necessária uma complementação hídrica, surgem diversas alternativas para o cultivo da cultura, como a utilização de água residuária, que pode ser utilizada por diversas propriedades ao longo do semiárido, principalmente através da agricultura familiar para o cultivo de cultura. Estudos vêm sendo conduzidos no Agreste do Estado, localizado no Distrito de Mutuca em PesqueiraPE, em uma vila com 150 residências e aproximadamente 750 pessoas, através de projeto de pesquisa e extensão, vinculados à Universidade Federal Rural de Pernambuco-UFRPE, na qual, são desenvolvidos na Unidade de Tratamento de Reuso e Manejo Hidroagrícola, com objetivo da utilização da água residuária, evitando a contaminação da nascente do Rio Ipojuca e ao mesmo tempo possibilitando a aplicação da água ao cultivo da moringa, contribuindo para sustentabilidade e proteção ambiental nessas áreas (Santos, 2015).

\section{Conclusão}

Com a realização do zoneamento foi possível identificar as potencialidades e restrições existentes a exploração comercial da cultura da moringa no Estado de Pernambuco, de modo a auxiliar produtores na tomada de decisão sobre o sistema de produção, bem como em ações políticas de financiamento e investimentos para a expansão da cultura, considerando os efeitos de mudanças climáticas no Estado.

A partir do zoneamento da aptidão climática, identificaram-se que uma pequena área na Zona da Mata e na Região Metropolitana apresentam inaptidão para o cultivo da moringa, devido ao excesso de chuva.

A elevação da temperatura para o Estado de Pernambuco, de acordo com as projeções de mudanças climáticas futuras, promoverá maiores áreas sem restrição térmica, possibilitando a maior expressão do potencial de produção da cultura.

\section{Agradecimentos}

À Financiadora de Estudos e Projetos (FINEP), à Fundação de Amparo à Ciência e Tecnologia do Estado de Pernambuco (FACEPE) e ao Conselho Nacional de Desenvolvimento Científico e Tecnológico (CNPq), pelo apoio ao desenvolvimento da pesquisa.

\section{Referências}

ABRANTES, J. R. C. B. O.; LIMA, J. L. M. P.; MONTENEGRO, A. A. A. 2015. Desempenho da modelagem cinemática do escoamento superficial para chuvas intermitentes em solos com cobertura morta. Revista Brasileira de Engenharia Agrícola e Ambiental, v. 19, n. 1, p. 166-172.

BEZERRA, A. M. E.; MOMENTÉ, V. G.; MEDEIROS FILHO, S. 2004. Germinação de 
sementes e desenvolvimento de plântulas de moringa (Moringa oleifera Lam.) em função do peso da semente e do tipo de substrato. Horticultura Brasileira, v. 22, n. 2, p. 295-299.

BORGES, T. K. S.; MONTENEGRO, A. A. A.; SANTOS, T. E. M.; SILVA, D. D.; SILVA JUNIOR, V. P. 2014. Influência de práticas conservacionistas na umidade do solo e no cultivo do milho (Zea mays L.) em semiárido nordestino. Revista Brasileira de Ciência do Solo, v. 38, n. 6, p. $1862-1873$.

CÁCERES, A.; FREIRE, V.; GIRÓN, L. M.; AVILÉS, O.; PACHECO, G. 1991. Moringa oleifera (Moringaceae): etnobotânical studies in Guatemala. Economic Botany, v. 45, n. 4, p. 522523.

GARDIMAN JÚNIOR, B. S.; MAGALHÃES, I. A. L.; FREITAS, C. A. A.; CECÍLIO, R. A. 2012. Análise de técnicas de interpolação para espacialização da precipitação pluvial na bacia do rio Itapemirim (ES). Revista Ambiência, v. 8, n. 1 p. 61-71.

GASPARINI, K. A. C.; FONSECA, M. D. S.; PASTRO, M. S.; LACERDA, L. C.; SANTOS, A. R. 2015. Zoneamento agroclimático da cultura do açaí (Euterpe oleracea Mart.) para o estado do Espírito Santo. Revista Ciência Agronômica, v. 46, p. 707-717.

GAZA. 2007. Cidadão Solidário. Moringa: Folhas Nutritivas. Guarantina. Disponível em: http://www.cidadaosolidario.org.br/Moringa/Culti vodaMoringa.pdf. Acesso em: 03 fevereiro de 2017.

GUIRRA, F. 2008. Moringa e o biodiesel: 1001 Utilidades. Biodieselbr, v. 8, p. 30-34.

IPCC. 2013. Intergovernmental Panel on Climate Change. Climate change 2013: The Physical Science Basis. Contribution of working group I to the Fifth Assessment Report of the Intergovernmental Panel on Climate Change. Cambridge University Press, Cambridge, United Kingdom and New York, NY, USA. 1552p.

MONTEIRO, J. M. A. 2007. Plantio de oleaginosas por agricultores familiares do semiárido nordestino para produção de biodiesel como uma estratégia de mitigação e adaptação às mudanças climáticas. Tese de Doutorado, Universidade Federal do Rio de Janeiro, Rio de Janeiro-RJ. 302f.
MONTENEGRO, A. A. A.; ABRANTES, J. R. C. B. O.; DE LIMA, J. L. M. P.; SINGH, V.; SANTOS, T. E. 2013. Impact of Mulching on Soil and Water Dynamics under Intermittent Simulated Rainfall. Catena, v. 109, p. 139-149.

MORAIS, J. E. F., SILVA, T. G. F., BARBOSA, M. L., SILVA DINIZ, W. J., SOUZA, C. A. A., JARDIM, A. M. D. R. F., FERREIRA, J. M. S. 2015. Impactos das mudanças climáticas na demanda hídrica e duração do ciclo do sorgo forrageiro e feijão caupi no estado de Pernambuco. Revista Brasileira de Geografia Física, v. 8, p. 542-559.

NÓBREGA, R. S.; FARIAS, R. F. L.; SANTOS, C. A. C. 2015. Variabilidade temporal e espacial da precipitação pluviométrica em Pernambuco através de índices de extremos climáticos. Revista Brasileira deMeteorologia, v. 2, p. 171-180.

OLIVEIRA, I. C.; TEIXEIRA, E. M. B.; GONÇALVES, C. A. A.; PEREIRA, L. A. 2009. Avaliação centesimal da semente de Moringa oleifera Lam. In: II Seminário Iniciação Científica - IFTM, Campus Uberaba, MG.

PARROTTA, J. A. 1993. Moringa oleifera Lam. Resedá, horseradish tree. New Orleans, LA: U.S. Department of Agriculture, Forest Service, Southern Forest Experiment Station. 6p.

POSSAS, J.; CORREA, M. M.; MOURA, G. B. D. A.; LOPES, P. M.; CALDAS, A. M.; FONTES JÚNIOR, R. V. D. P. 2012. Zoneamento agroclimático para a cultura do pinhão-manso no Estado de Pernambuco. Revista Brasileira de Engenharia Agrícola e Ambiental, v. 16, n. 9, p. 993-998.

SANTANA, C. R.; PEREIRA, D. F.; ARAUJO, N. A.; CAVALCANTI, E. B.; SILVA, G. F. 2010. Caracterização Físico-Química da Moringa (Moringa oleifera Lam), Revista Brasileira de Produtos Agroindustriais, v. 12, n. 1, p. 55-60.

SANTOS, C. S. 2015. Conservação de solo e água em unidade de reuso do semiárido para proteção ambiental, produção agrícola e inclusão social. Dissertação de Mestrado, Universidade Federal Rural de Pernambuco, Recife, Pernambuco, Brasil. 131p.

SILVA, A. O.; MOURA, G. B. A.; KLAR, A. E. 2014. Classificação climática de Thornthwaite e sua aplicabilidade agroclimatológica nos 
diferentes regimes de precipitação em Pernambuco. Brazilian Journal of Irrigation and Drainage - Irriga, v. 19, n. 1, p. 46.

SILVA, T. G. F.; MOURA, M. S.; SÁ, I. I.; ZOLNIER, S.; TURCO, S. H.; SOUZA, L. S. 2010. Cenários de mudanças climáticas e seus impactos na produção leiteira em estados nordestinos. Revista Brasileira de Engenharia Agrícola e Ambiental, v. 14, n. 8, p. 863-870.

TEIXEIRA, C. M. L. L.; KIRSTEN, F. V.; TEIXEIRA, P.C.N. 2012. Evaluation of Moringa oleifera seed flour as a flocculating agent for potential biodiesel producer microalgae. Journal of Applied Phycology, v. 24, p. 557-563. 